\title{
Proton stereotactic body radiation therapy for liver metastases- results of 5-year experience for 81 hepatic lesions
}

\author{
Alex R. Coffman ${ }^{1}$, Daniel C. Sufficool ${ }^{2}$, Joseph I. Kang ${ }^{1}$, Chung-Tsen Hsueh ${ }^{3}$, Sasha Swenson ${ }^{4}$, \\ Patrick Q. McGee ${ }^{4}$, Gayathri Nagaraj ${ }^{3}$, Baldev Patyal ${ }^{1}$, Mark E. Reeves ${ }^{5}$, Jerry D. Slater ${ }^{1}$, Gary Y. Yang ${ }^{1}$ \\ ${ }^{1}$ Department of Radiation Oncology, Loma Linda University Medical Center, Loma Linda, CA, USA; ${ }^{2}$ Department of Radiation Oncology, \\ Kettering Health Network, Kettering, OH, USA; ${ }^{3}$ Department of Medical Oncology, Loma Linda University Medical Center, Loma Linda, CA, \\ USA; ${ }^{4}$ Loma Linda University School of Medicine, Loma Linda, CA, USA; ${ }^{5}$ Department of Surgical Oncology, Loma Linda University Medical \\ Center, Loma Linda, CA, USA \\ Contributions: (I) Conception and design: GY Yang; (II) Administrative support: B Patyal, JD Slater, GY Yang; (III) Provision of study materials or \\ patients: CT Hsueh, G Nagaraj, ME Reeves; (IV) Collection and assembly of data: AR Coffman, GY Yang; (V) Data analysis and interpretation: AR \\ Coffman, GY Yang; (VI) Manuscript writing: All authors; (VII) Final approval of manuscript: All authors. \\ Correspondence to: Alex R. Coffman, MD. Department of Radiation Oncology, Loma Linda University Medical Center, 11234 Anderson Street, Suite \\ B121, Loma Linda, CA 92354, USA. Email: acoffman@llu.edu.
}

\begin{abstract}
Background: To report on our institutional experience using Proton stereotactic body radiation therapy (SBRT) for patients with liver metastases.

Methods: All patients with liver metastases treated with Proton SBRT between September 2012 and December 2017 were retrospectively analyzed. Local control (LC) and overall survival (OS) were estimated using the Kaplan-Meier method calculated from the time of completion of Proton SBRT. LC was defined according to Response Evaluation Criteria in Solid Tumors (RECIST) guidelines (version 1.1). Toxicity was graded according to Common Terminology Criteria for Adverse Events (CTCAE) version 4.0.

Results: Forty-six patients with 81 lesions were treated with Proton SBRT. The median age was 65.5 years old (range, 33-86 years) and the median follow up was 15 months (range, 1-54 months). The median size of the gross tumor volume (GTV) was $2.5 \mathrm{~cm}$ (range, 0.7-8.9 cm). Two or more lesions were treated in $56.5 \%$ of patients, with one patient receiving treatment to a total of five lesions. There were 37 lesions treated with a biologically effective dose (BED) $\leq 60,9$ lesions with a BED of 61-80, 22 lesions with a BED of 81-100, and 13 lesions with a BED >100. The 1-year and 2-year LC for all lesions was $92.5 \%$ (95\% CI, $82.7 \%$ to $96.8 \%$ ). The grade 1 and grade 2 toxicity rates were $37 \%$ and $6.5 \%$, respectively. There were no grade 3 or higher toxicities and no cases of radiation-induced liver disease (RILD).

Conclusions: Proton SBRT for the treatment of liver metastases has promising LC rates with the ability to safely treat multiple liver metastases. Accrual continues for our phase II trial treating liver metastases with Proton SBRT to 60 GyE (Gray equivalent) in 3 fractions.
\end{abstract}

Keywords: Liver metastases; proton therapy; stereotactic body radiation therapy (SBRT)

Submitted Oct 06, 2020. Accepted for publication Feb 01, 2021.

doi: 10.21037/jgo-20-424

View this article at: http://dx.doi.org/10.21037/jgo-20-424

\section{Introduction}

The liver is a common organ for metastatic disease involvement in many solid malignancies including gastrointestinal, breast, and lung cancer. Among patients with colorectal cancer (CRC), approximately $50 \%$ will develop metastatic disease to the liver $(1,2)$. Aggressive local treatment to these lesions can increase survival in certain scenarios. In patients with oligometastatic disease to the liver, there is even a possibility of long-term disease control. 
Hepatic resection is the standard of care in these situations with five-year survival rates generally around $25-45 \%$ (3-7). There is also data on surgical resection of liver metastases in patients with non-CRC primaries (8-10). Unfortunately, a majority of patients are not candidate for resection (11). Therefore, safe and effective treatments for metastatic hepatic disease are an important clinical need.

Other local therapy options for patients that develop liver metastases include radiofrequency ablation (RFA), cryotherapy, transarterial chemoembolization (TACE), yttrium-90 (Y90), and stereotactic body radiation therapy (SBRT). High rates of local control (LC) of hepatic disease appears to be a key determinant of overall survival (OS) $(3,5,10,12,13)$. For example, Aloia et al. showed RFA was associated with a seven-fold increased risk of local failure and three-fold increased risk of death compared to resection despite similar rates of intrahepatic and extrahepatic failure among patients with solitary hepatic disease from CRC (13). A recent meta-analysis demonstrated superior LC for SBRT compared to RFA in the treatment of liver metastases (14). Along with good LC, the ideal treatment would also be associated with minimal morbidity. SBRT has been shown to be safe in dose escalation studies (15-18) with excellent control rates above $90 \%$ with biologically effective doses (BED) $>100 \mathrm{~Gy}_{10}$ (19). Rusthoven et al. reported a 2-year LC rate of $94 \%$ for 38 lesions treated with 60 Gy in 3 fractions, with a 2-year LC rate of $100 \%$ for lesions $3 \mathrm{~cm}$ or less (16). SBRT also has the advantage over other local therapies of being non-invasive.

Proton therapy's unique characteristics of the Bragg peak and a lack of exit dose can potentially provide additional benefits for Proton SBRT treatment of liver metastases. Multiple studies illustrate a dosimetric advantage of proton therapy compared to photon-based therapy in liver radiotherapy resulting in higher volumes of liver irradiation with photon therapy (20-22). Subsequent courses of treatment may be limited by prior liver radiation exposure with photon therapy. Proton SBRT limits the integral dose to the liver making subsequent courses of treatment more feasible. Joo et al. reported a $59 \%$ out-offield recurrence rate in the liver for these patients showing the high probability of additional courses of liver directed therapy (23). Proton-based radiation treatment to the liver has also been shown to be safe in patients with hepatocellular carcinoma (HCC), who most often have underlying cirrhosis (24-26). Only one phase II Proton SBRT liver metastases study is published to date showing lower rates of LC compared to contemporary series (27).
However, the authors note that this is likely related to a lower BED. A phase I dose escalation trial showed Proton SBRT to be safe and well tolerated with the maximal tolerated dose not reached at a dose of $60 \mathrm{GyE}$ (Gray equivalent) (28).

In this study we analyzed all patients that were treated with Proton SBRT for liver metastases at our institution in the initial five years. The primary objective was to identify the LC rate of Proton SBRT in patients with metastatic liver disease. Secondary objectives include evaluation of OS and toxicity. We present the following article in accordance with the Strengthening the Reporting of Observational studies in Epidemiology (STROBE) reporting checklist. (available at: http://dx.doi.org/10.21037/jgo-20-424).

\section{Methods}

A retrospective analysis was completed of all 46 patients with 81 liver metastases that were treated at our institution between September 2012 and December 2017. The fractionation regimens, LC rates, OS, and toxicity were evaluated. Potential bias was reduced by analyzing every patient that was treated for liver metastases over this time period.

\section{Fractionation schemes and dose constraints}

Some patients were treated on our institutional phase I/ II clinical trial (NCT01697371) with a 3-fraction regimen with dose escalation of 36,48 , and $60 \mathrm{GyE}$. The dose in proton therapy is defined in terms of effective dose, which is the physical dose in Gray multiplied by a relative biological effectiveness (RBE) of 1.1, which has been validated in the clinic (29). The $60 \mathrm{GyE}$ regimen had no dose limiting toxicity and is used for the phase II trial with dose constraints specified by protocol (28). Other patients who did not meet eligibility criteria for the phase II trial were treated in 3 or 5 fractions. For the five-fraction regimen, common dose prescriptions included 30 and 50 GyE (range, 30-50 GyE). Dose constraints were adapted from the NRG BR001 protocol (NCT02206334). The liver constraint for three fractions is V17 $<700 \mathrm{cc}$ and for five fractions is V21<700 cc. The stomach constraint for three fractions is V30 $<0.03 \mathrm{cc}$ and V22.5 $<10 \mathrm{cc}$ and for five fractions is V35<0.5 cc and V26.5 $<5 \mathrm{cc}$. The small bowel constraint for three fractions is $\mathrm{V} 34.5<0.03 \mathrm{cc}$ and $\mathrm{V} 24<20 \mathrm{cc}$ and for five fractions is $\mathrm{V} 40<0.03 \mathrm{cc}$ and V28.5 $<20$ cc. 


\section{Immobilization and tumor volume definition}

Patients underwent computed tomography (CT) simulation with intravenous contrast with a full body pod for immobilization, as previously described (30). Tumor motion was accounted for by either four-dimensional CT (4DCT) or deep inspiration breath hold (DIBH) (SDX, Qfix, Avondale, PA).

For patients using 4DCT, an internal target volume (ITV) was defined to encompass the enhancing or hypoattenuating lesion on the maximum intensity projection (MIP) scan (31). For patients using SDX, gross tumor volume (GTV) was defined to encompass the enhancing or hypoattenuating lesion on the CT scan with contrast. The GTV was then expanded by a $5 \mathrm{~mm}$ radial and $10 \mathrm{~mm}$ craniocaudal margin to create the ITV (16). Additionally, each passively scattered proton field was optimized to account for proton beam modulation, Bragg peak, depth dose, uncertainty, and energy optimization. A multi-beam, computer-generated treatment plan was created to deliver the prescription dose in 3 or 5 equal fractions.

\section{Statistical analysis}

LC was defined according to Response Evaluation Criteria in Solid Tumors (RECIST) guidelines (version 1.1) (32). OS was assessed as the time period between completion of the last treatment and either death by any cause, or censored at last known follow up. The Kaplan-Meier method was used to estimate LC and OS. Statistical analysis was completed using Python (33).

\section{Toxicity}

Acute toxicity was graded according to Common Terminology Criteria for Adverse Events (CTCAE) version 4.0. Specific factors of interest included evaluation for radiation-induced liver disease (RILD). RILD is a clinical syndrome of anicteric ascites, hepatomegaly, and elevation of alkaline phosphatase (ALP) relative to other transaminases that may occur 2 weeks to 3 months following radiation to the liver. Grade 3 ALP ( $>5$ times the upper limit of normal) with ascites not due to cancer progression was considered RILD. Other evaluated toxicity included toxicity to any related organ system within 90 days from the start of treatment.

\section{Follow up}

Patients were planned to be evaluated once during the treatment course, at 4 weeks after treatment, and at 3 months after treatment. Evaluation included physical examination, laboratory values, follow-up imaging (starting at 3 months), and a detailed account of possible symptoms. Any observed toxicity within 90 days of treatment was recorded as acute toxicity. Regular follow-up was planned to continue at 3-month intervals to evaluate treatment response. In cases of missing follow-up data, patients were censored when last known to be alive.

\section{Ethical statement}

The study was conducted in accordance with the Declaration of Helsinki (as revised in 2013). The study was approved by the Institutional Review Board (IRB) of Loma Linda University Health (IRB\#5200383) and informed consent was taken from all the patients.

\section{Results}

\section{Patient population}

Forty-six patients with 81 total lesions were treated with Proton SBRT. Patient characteristics are listed in Table 1. The median age was 65.5 years old (range, 33-86 years). The median follow up was 15 months (range, 1-54 months). Half of the patients were male $(n=23)$ and half were female $(n=23)$ and $87 \%$ of patients had an Eastern Cooperative Oncology Group (ECOG) score of 0 . The primary site of disease was colorectal in $50.0 \%$ of cases. Extrahepatic disease was present in $43.5 \%$ of patients with $19.6 \%$ receiving some sort of prior local therapy to the liver before Proton SBRT. Two or more lesions were treated in $56.5 \%$ of patients, with one patient receiving treatment to a total of five lesions. The median size of the GTV was $2.5 \mathrm{~cm}$ (range, $0.7-8.9 \mathrm{~cm}$ ) with a median volume of $4.07 \mathrm{cc}$ (range, $0.23-125.58 \mathrm{cc}$ ). A majority of patients (89.1\%) underwent 4DCT for immobilization.

\section{Fractionation schemes and BED}

For the three-fraction treatment, doses included $27 \mathrm{GyE}$ (27.8\%), 30 GyE (16.7\%), 36 GyE (19.4\%), 48 Gy (19.4\%), 
Table 1 Patient and tumor characteristics

\begin{tabular}{|c|c|}
\hline Characteristics & Number \\
\hline Patients & 46 \\
\hline Lesions & 81 \\
\hline \multicolumn{2}{|l|}{ Age (years) } \\
\hline Median & 65.5 \\
\hline Range & $33.7-86.7$ \\
\hline \multicolumn{2}{|l|}{ Sex, n (\%) } \\
\hline Male & $23(50.0)$ \\
\hline Female & $23(50.0)$ \\
\hline \multicolumn{2}{|l|}{ ECOG, n (\%) } \\
\hline 0 & $40(87.0)$ \\
\hline 1 & $4(8.7)$ \\
\hline 2 & $2(4.3)$ \\
\hline \multicolumn{2}{|c|}{ Primary site, n (\%) } \\
\hline Colon & $15(32.6)$ \\
\hline Rectum & $8(17.4)$ \\
\hline Breast & $6(13.0)$ \\
\hline Endometrial & $3(6.5)$ \\
\hline Pancreas & $2(4.3)$ \\
\hline NSCLC & $2(4.3)$ \\
\hline Other & $10(21.7)$ \\
\hline \multicolumn{2}{|c|}{ Number of lesions per patient, $n(\%)$} \\
\hline 1 & $20(43.5)$ \\
\hline 2 & $15(32.6)$ \\
\hline 3 & $6(13.0)$ \\
\hline 4 & $4(8.7)$ \\
\hline 5 & $1(2.2)$ \\
\hline \multicolumn{2}{|l|}{ GTV size $(\mathrm{cm})$} \\
\hline Median & 2.5 \\
\hline Range & $0.7-8.9$ \\
\hline \multicolumn{2}{|l|}{ GTV volume (cc) } \\
\hline Median & 4.07 \\
\hline Range & $0.23-125.58$ \\
\hline \multicolumn{2}{|c|}{ Extrahepatic disease, $\mathrm{n}(\%)$} \\
\hline Yes & $20(43.5)$ \\
\hline No & $26(56.5)$ \\
\hline \multicolumn{2}{|c|}{ Prior local therapy, n (\%) } \\
\hline Yes & $9(19.6)$ \\
\hline No & $37(80.4)$ \\
\hline
\end{tabular}

ECOG, Eastern Collaborative Oncology Group; NSCLC, nonsmall cell lung cancer; GTV, gross tumor volume. and $60 \mathrm{GyE}(16.7 \%)$. For the five-fraction treatment, common doses included $30 \mathrm{GyE}(46.7 \%)$ and $50 \mathrm{GyE}$ (40\%). There was a large range of BEDs that were used for the 81 lesions that were treated. The BED was calculated assuming an alpha/beta ratio of 10 . There were 37 lesions treated with a BED $\leq 60,9$ lesions with a BED of 61-80, 22 lesions with a BED of 81-100, and 13 lesions with a BED $>100$. A common prescription in the low BED group was $30 \mathrm{GyE}$ in 5 fractions, which was often used to meet the dose constraint for small bowel or stomach when it was in close proximity to the target volume.

\section{Clinical outcomes}

LC at 6 months was $97.3 \%$ (95\% CI, $89.8 \%$ to $99.3 \%$ ) for the 81 lesions evaluated. LC at 1 year and 2 years was $92.5 \%$ (95\% CI, $82.7 \%$ to $96.8 \%$ ). LC at 3 years was $71.9 \%$ (95\% CI, $53.2 \%$ to $84.2 \%$ ) (see Figure 1). The longest duration of LC was in a patient who had 54 months followup, but there were also 10 other patients that had continued LC $>45$ months after treatment.

LC for patients that had a BED of $\geq 100$ is illustrated in Figure 2. In these 13 lesions, the LC at 6 months was $90.0 \%$ (95\% CI, $65.3 \%$ to $97.4 \%$ ). The LC at 1 year was $84.0 \%$ (95\% CI, $57.8 \%$ to $94.6 \%$ ). Three of the 13 patients experienced local failure after the 7 -month mark, after which the curve remains flat. Two of these lesions were large measuring 5.4 and $6.9 \mathrm{~cm}$. The third lesion was a $1.5 \mathrm{~cm}$ lesion from a primary rectal cancer that received $48 \mathrm{GyE}$ in 3 fractions. This patient also had a $1.3 \mathrm{~cm}$ lesion that was treated with $36 \mathrm{GyE}$ in 3 fractions, which also experienced treatment failure at the same time point.

LC was also analyzed for 37 lesions that received a BED of $\leq 60$. The median tumor size among these 37 lesions was $1.8 \mathrm{~cm}$. The LC at 6 months was $97.1 \%$ (95\% CI, $81.4 \%$ to 99.6\%). The 1 year and 2-year LC rates were $90.4 \%$ (95\% CI, $73.1 \%$ to $96.8 \%$ ). The 3 -year LC rate was $64.7 \%$ (95\% CI, $33.7 \%$ to $84.1 \%)$. This data is not presented in graphical form.

The median survival for the 46 patients was 30.0 months. The 1 -year OS was $68.1 \%$ (95\% CI, $52.1 \%$ to $79.8 \%$ ), 2 -year OS was $58.1 \%$ (95\% CI, $41.8 \%$ to $72.1 \%$ ), 3-year OS was $43.8 \%$ (95\% CI, $27.3 \%$ to $58.1 \%$ ), and 4 -year OS was $20.1 \%$ (95\% CI, $6.6 \%$ to $40.2 \%$ ) (see Figure 3). At the time of analysis eight patients were still alive.

\section{Toxicity}

Grade 1 toxicity was experienced in 17 of $46(37.0 \%)$ 


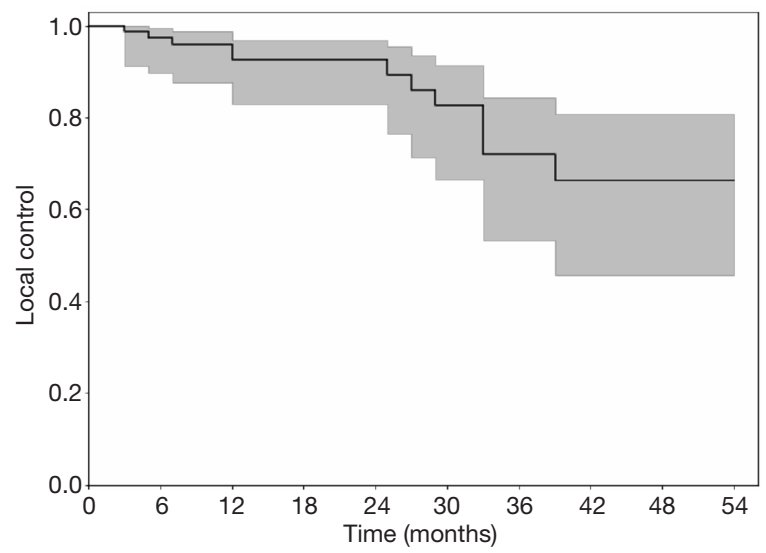

Figure 1 Local control using Kaplan-Meier method with $95 \%$ confidence bounds.

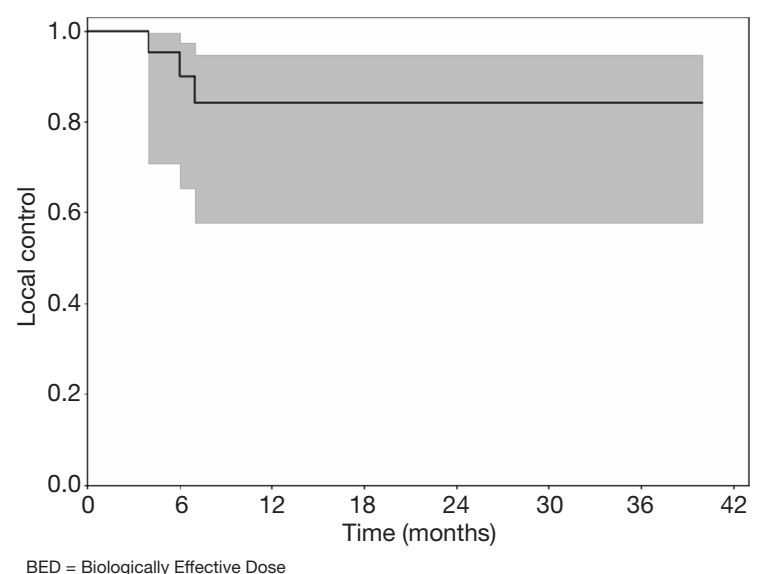

Figure 2 Local control using Kaplan-Meier method with 95\% confidence bounds for only patients with a BED $\geq 100$. BED, biologically effective dose.

patients and grade 2 toxicity was experienced in 3 of 46 patients $(6.5 \%)$. No grade 3 or higher toxicities were reported and no cases of RILD were detected. The median percentage of liver that received no radiation was $59.9 \%$ (range, $25.0-91.7 \%$ ). Among the 17 patients that experienced a grade 1 toxicity, 10 patients $(58.8 \%)$ experienced elevated liver enzymes, four patients $(23.5 \%)$ experienced fatigue, one patient $(5.9 \%)$ experienced skin hyperpigmentation, and two patients (11.8\%) experienced both elevated liver enzymes and skin hyperpigmentation. Among the three patients that experienced grade 2 toxicity, two patients experienced fatigue that was not relieved by rest, which limited instrumental activities of daily living

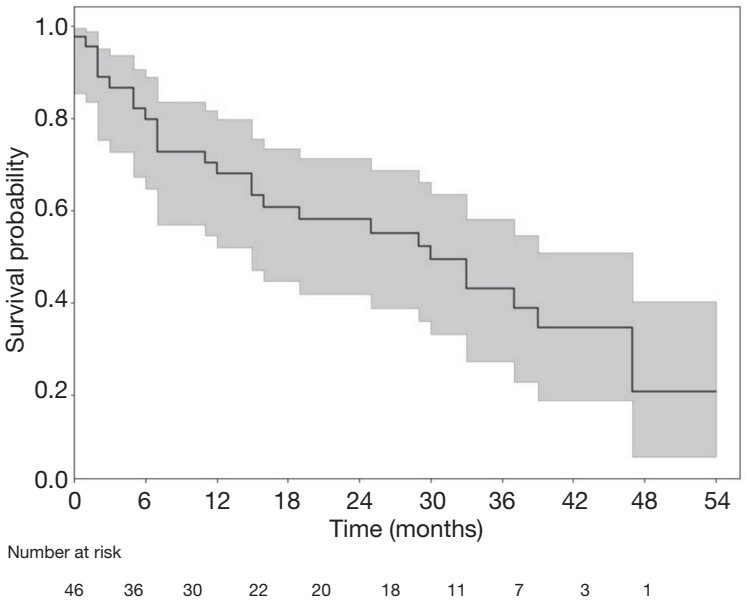

Figure 3 Overall survival using Kaplan-Meier method with $95 \%$ confidence bounds.

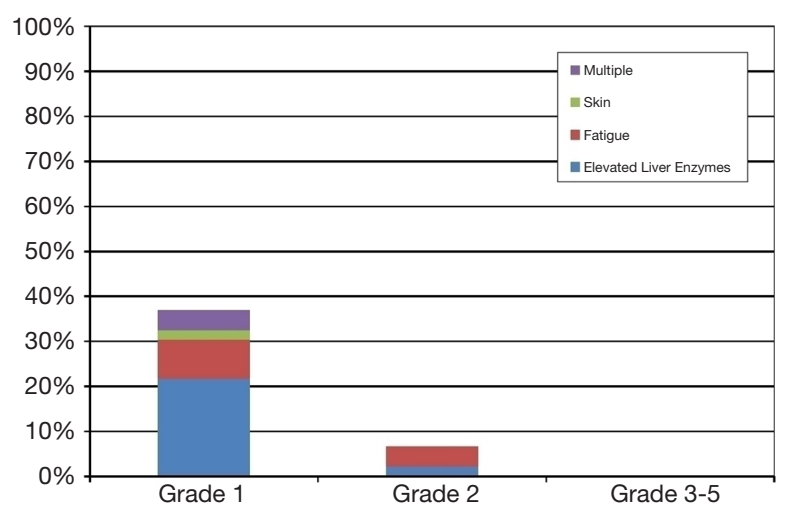

Figure 4 Acute toxicity using Common Terminology Criteria for Adverse Events (CTCAE) version 4.03.

(ADL) and the third patient experienced a greater increase in liver enzymes (see Figure 4).

\section{Discussion}

In this study, we report the results of our 5 -year institutional experience treating liver metastases with Proton SBRT. The 2 -year LC for all 81 lesions treated was $92.5 \%$. There was no grade 3 or higher toxicity and no incidences of RILD.

The LC was high despite a large number of patients treated with a low BED. A 2-year LC of $90.4 \%$ for 37 lesions treated with a BED $\leq 60$ was unexpected. Smaller tumor size in this cohort (median $1.8 \mathrm{~cm}$ ) is a possible contributor. It is also possible that a lower BED can delay the disease progression, but may not lead to a durable 
response, as the 3 -year LC rate dropped to $64.7 \%$. The 2-year LC rate was $84.0 \%$ for 13 lesions treated with a BED $\geq 100$. The lower LC compared to the lower BED cohort may be explained by two large lesions that had early local failure. Liver metastases greater than $5 \mathrm{~cm}$ are typically excluded from most SBRT protocols. The curve remains flat after these early failures suggesting a durable response for the other lesions treated with a high BED.

LC, the primary end point in this study, was similar to photon-based SBRT as expected (16,19). However, the main advantage of Proton SBRT is the reduced integral liver dose providing the option of safely delivering multiple courses of treatment. When patients are considered for hepatic resection, the limit for safe resection is generally $20-30 \%$ of remnant liver (34). The median percentage of liver that received no radiation dose from our data was $59.9 \%$, despite a majority of patients receiving two or more courses of treatment. This is significant as the out-of-field recurrence rate in the liver can be high (23). Therefore, preserving the possibility for additional courses of SBRT in the future is a major benefit of Proton SBRT.

The MS was 30 months, which is high considering almost half of patients had extrahepatic disease. This partially reflects the efficacy of systemic treatment. In general, patients were considered for Proton SBRT to new or progressing liver metastases if the extrahepatic disease was stable on systemic treatment. The 4-year OS was $20.1 \%$ and eight patients were still alive at the time of this analysis. Since LC of liver metastases appears to be associated with OS $(3,5,10,12,13)$, SBRT offers the possibility of long-term survival in some patients with oligometastatic disease even if they are not candidates for hepatic resection.

Proton SBRT appears to be safe with no patients experiencing grade 3 or higher toxicity or RILD. This is significant considering $56.5 \%$ of patients completed treatment to two or more liver metastases. Routine use of DIBH for those who can tolerate it, as opposed to 4DCT, can further reduce integral liver dose, potentially increasing the benefit of Proton SBRT even further.

Our phase I dose escalation study established the safety of the $60 \mathrm{GyE}$ in 3 fraction dose regimen (28). This is the preferred regimen for treatment now as long as there are no dose-limiting adjacent structures. For lesions adjacent to chest wall or rib, we use $50 \mathrm{GyE}$ in 5 fractions to reduce the risk of chest wall pain or rib fracture (35). We use $30 \mathrm{GyE}$ in 5 fractions in patients that have liver metastasis near stomach or bowel. However, an alternative local treatment modality should be considered, if possible, as durable LC is expected to be low in these situations.

The limitations of this study include the common limitations that apply to any observational study. All patients treated with Proton SBRT for liver metastases over this time period were analyzed to reduce selection bias. However, patients that received Proton SBRT for liver metastases were likely carefully selected by the referring provider based on performance status and ability to tolerate treatment. These results are likely not generalizable to all patients with liver metastases, especially patients with poor performance status or inadequate hepatic reserve.

In conclusion, Proton SBRT has high LC rates with an excellent toxicity profile for treatment of liver metastases even when a majority of patients received multiple courses of treatment. We continue to accrue patients for our Phase II study treating liver metastases with Proton SBRT to $60 \mathrm{GyE}$ in 3 fractions.

\section{Acknowledgments}

Sandra C. Teichman for her assistance in the IRB approval process.

Funding: None.

\section{Footnote}

Reporting Checklist: The authors have completed the STROBE reporting checklist. Available at: http://dx.doi. org/10.21037/jgo-20-424

Data Sharing Statement: Available at: http://dx.doi. org/10.21037/jgo-20-424

Peer Review File: Available at: http://dx.doi.org/10.21037/ jgo-20-424

Conflicts of Interest: All authors have completed the ICMJE uniform disclosure form (available at: http://dx.doi. org/10.21037/jgo-20-424). The authors have no conflicts of interest to declare.

Ethical Statement: The authors are accountable for all aspects of the work in ensuring that questions related to the accuracy or integrity of any part of the work are appropriately investigated and resolved. The study was conducted in accordance with the Declaration of Helsinki (as revised in 2013). The study was approved by the Institutional Review Board (IRB) of Loma Linda University 
Health (IRB\#5200383) and informed consent was taken from all the patients.

Open Access Statement: This is an Open Access article distributed in accordance with the Creative Commons Attribution-NonCommercial-NoDerivs 4.0 International License (CC BY-NC-ND 4.0), which permits the noncommercial replication and distribution of the article with the strict proviso that no changes or edits are made and the original work is properly cited (including links to both the formal publication through the relevant DOI and the license). See: https://creativecommons.org/licenses/by-nc-nd/4.0/.

\section{References}

1. Welch JP, Donaldson GA. The clinical correlation of an autopsy study of recurrent colorectal cancer. Ann Surg 1979;189:496-502.

2. Geoghegan JG, Scheele J. Treatment of colorectal liver metastases. Br J Surg 1999;86:158-69.

3. Rees M, Tekkis PP, Welsh FK, et al. Evaluation of longterm survival after hepatic resection for metastatic colorectal cancer: a multifactorial model of 929 patients. Ann Surg 2008;247:125-35.

4. Fong Y, Fortner J, Sun RL, et al. Clinical score for predicting recurrence after hepatic resection for metastatic colorectal cancer: analysis of 1001 consecutive cases. Ann Surg 1999;230:309-18; discussion 318-21.

5. Wei AC, Greig PD, Grant D, et al. Survival after hepatic resection for colorectal metastases: a 10-year experience. Ann Surg Oncol 2006;13:668-76.

6. Robertson DJ, Stukel TA, Gottlieb DJ, et al. Survival after hepatic resection of colorectal cancer metastases: a national experience. Cancer 2009;115:752-9.

7. Morris EJ, Forman D, Thomas JD, et al. Surgical management and outcomes of colorectal cancer liver metastases. Br J Surg 2010;97:1110-8.

8. Takemura N, Saiura A. Role of surgical resection for noncolorectal non-neuroendocrine liver metastases. World J Hepatol 2017;9:242-51.

9. Lendoire J, Moro M, Andriani O, et al. Liver resection for non-colorectal, non-neuroendocrine metastases: analysis of a multicenter study from Argentina. HPB (Oxford) 2007;9:435-9.

10. Chua TC, Saxena A, Liauw W, et al. Hepatic resection for metastatic breast cancer: a systematic review. Eur J Cancer 2011;47:2282-90.

11. Scheele J, Stangl R, Altendorf-Hofmann A. Hepatic metastases from colorectal carcinoma: impact of surgical resection on the natural history. Br J Surg 1990;77:1241-6.

12. Chang DT, Swaminath A, Kozak M, et al. Stereotactic body radiotherapy for colorectal liver metastases: a pooled analysis. Cancer 2011;117:4060-9.

13. Aloia TA, Vauthey JN, Loyer EM, et al. Solitary colorectal liver metastasis: resection determines outcome. Arch Surg 2006; 141:460-6; discussion 466-7.

14. Lee J, Shin IS, Yoon WS, et al. Comparisons between radiofrequency ablation and stereotactic body radiotherapy for liver malignancies: Meta-analyses and a systematic review. Radiother Oncol 2020;145:63-70.

15. Schefter TE, Kavanagh BD, Timmerman RD, et al. A phase I trial of stereotactic body radiation therapy (SBRT) for liver metastases. Int J Radiat Oncol Biol Phys 2005;62:1371-8.

16. Rusthoven KE, Kavanagh BD, Cardenes H, et al. Multiinstitutional phase I/II trial of stereotactic body radiation therapy for liver metastases. J Clin Oncol 2009;27:1572-8.

17. Goodman KA, Wiegner EA, Maturen KE, et al. Doseescalation study of single-fraction stereotactic body radiotherapy for liver malignancies. Int $\mathrm{J}$ Radiat Oncol Biol Phys 2010;78:486-93.

18. Rule W, Timmerman R, Tong L, et al. Phase I doseescalation study of stereotactic body radiotherapy in patients with hepatic metastases. Ann Surg Oncol 2011;18:1081-7.

19. Ohri N, Tomé WA, Méndez Romero A, et al. Local Control After Stereotactic Body Radiation Therapy for Liver Tumors. Int J Radiat Oncol Biol Phys 2021;110:188-95.

20. Wang X, Krishnan S, Zhang X, et al. Proton radiotherapy for liver tumors: dosimetric advantages over photon plans. Med Dosim 2008;33:259-67.

21. Petersen JB, Lassen Y, Hansen AT, et al. Normal liver tissue sparing by intensity-modulated proton stereotactic body radiotherapy for solitary liver tumours. Acta Oncol 2011;50:823-8.

22. Chuong M, Kaiser A, Molitoris J, et al. Proton beam therapy for liver cancers. J Gastrointest Oncol 2020;11:157-65.

23. Joo JH, Park JH, Kim JC, et al. Local Control Outcomes Using Stereotactic Body Radiation Therapy for Liver Metastases From Colorectal Cancer. Int J Radiat Oncol Biol Phys 2017;99:876-83.

24. Nakayama H, Sugahara S, Tokita M, et al. Proton beam therapy for hepatocellular carcinoma: the University of Tsukuba experience. Cancer 2009;115:5499-506. 
25. Fukumitsu N, Sugahara S, Nakayama H, et al. A prospective study of hypofractionated proton beam therapy for patients with hepatocellular carcinoma. Int J Radiat Oncol Biol Phys 2009;74:831-6.

26. Bush DA, Kayali Z, Grove R, et al. The safety and efficacy of high-dose proton beam radiotherapy for hepatocellular carcinoma: a phase 2 prospective trial. Cancer 2011;117:3053-9.

27. Hong TS, Wo JY, Borger DR, et al. Phase II Study of Proton-Based Stereotactic Body Radiation Therapy for Liver Metastases: Importance of Tumor Genotype. J Natl Cancer Inst 2017. doi: 10.1093/jnci/djx031.

28. Kang JI, Sufficool DC, Hsueh CT, et al. A phase I trial of Proton stereotactic body radiation therapy for liver metastases. J Gastrointest Oncol 2019;10:112-7.

29. Paganetti H, Niemierko A, Ancukiewicz M, et al. Relative biological effectiveness (RBE) values for proton beam therapy. Int J Radiat Oncol Biol Phys 2002;53:407-21.

30. Wroe AJ, Bush DA, Schulte RW, et al. Clinical

Cite this article as: Coffman AR, Sufficool DC, Kang JI, Hsueh CT, Swenson S, McGee PQ, Nagaraj G, Patyal B, Reeves ME, Slater JD, Yang GY. Proton stereotactic body radiation therapy for liver metastases-results of 5-year experience for 81 hepatic lesions. J Gastrointest Oncol 2021;12(4):1753-1760. doi: 10.21037/jgo-20-424 immobilization techniques for proton therapy. Technol Cancer Res Treat 2015;14:71-9.

31. Knecht ML, Wang N, Vassantachart A, et al. Individualized 4-dimensional computed tomography proton treatment for pancreatic tumors. J Gastrointest Oncol 2017;8:675-82.

32. Eisenhauer EA, Therasse P, Bogaerts J, et al. New response evaluation criteria in solid tumours: revised RECIST guideline (version 1.1). Eur J Cancer 2009;45:228-47.

33. Rossum GV, Drake FL. Python 3 Reference Manual: CreateSpace; 2009.

34. Guglielmi A, Ruzzenente A, Conci S, et al. How much remnant is enough in liver resection? Dig Surg 2012;29:6-17.

35. Stephans KL, Djemil T, Reddy CA, et al. A comparison of two stereotactic body radiation fractionation schedules for medically inoperable stage I non-small cell lung cancer: the Cleveland Clinic experience. J Thorac Oncol 2009;4:976-82. 